\title{
Glial cell activity is maintained during prolonged inflammatory
} challenge in rats

B.C. Borges, R. Rorato, J. Antunes-Rodrigues and L.L.K. Elias

The Brazilian Journal of Medical and Biological Research is partially financed by

\section{贵CNPq} Conselfo Nacional de Des
Cientifico e Tecroologico
Ministério da Ciência e Tecnologia

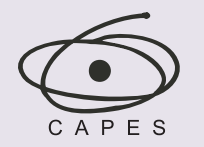

Ministério da Educação

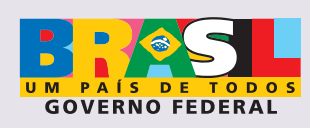

Institutional Sponsors

sue $\mathbb{R}_{0}$
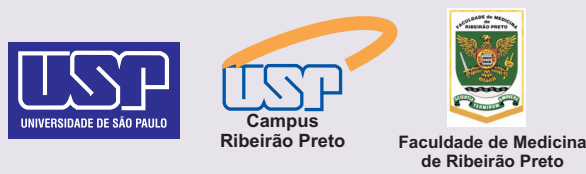

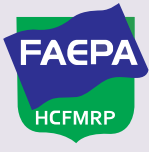

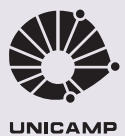

UNICAMP

\section{Ф SHIMADZU}

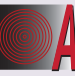

Afl

Associaçando Fundo
de Incentivo
à Pesquisa

Explore High - Performance MS Orbitrap Technology

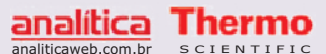




\title{
Glial cell activity is maintained during prolonged inflammatory challenge in rats
}

\author{
B.C. Borges, R. Rorato, J. Antunes-Rodrigues and L.L.K. Elias \\ Departamento de Fisiologia, Faculdade de Medicina de Ribeirão Preto, \\ Universidade de São Paulo, Ribeirão Preto, SP, Brasil
}

\begin{abstract}
We evaluated the expression of glial fibrillary acidic protein (GFAP), glutamine synthetase (GS), ionized calcium binding adaptor protein-1 (Iba-1), and ferritin in rats after single or repeated lipopolysaccharide (LPS) treatment, which is known to induce endotoxin tolerance and glial activation. Male Wistar rats $(200-250 \mathrm{~g})$ received ip injections of LPS $(100 \mu \mathrm{g} / \mathrm{kg}) \mathrm{or}$ saline for 6 days: 6 saline $(\mathrm{N}=5), 5$ saline +1 LPS $(N=6)$ and 6 LPS $(N=6)$. After the sixth injection, the rats were perfused and the brains were collected for immunohistochemistry. After a single LPS dose, the number of GFAP-positive cells increased in the hypothalamic arcuate nucleus (ARC; 1 LPS: $35.6 \pm 1.4$ vs control: $23.1 \pm 2.5$ ) and hippocampus (1 LPS: $165.0 \pm 3.0$ vs control: $137.5 \pm 2.5)$, and interestingly, 6 LPS injections further increased GFAP expression in these regions $(A R C=52.5 \pm 4.3$; hippocampus $=182.2 \pm 4.1$ ). We found a higher GS expression only in the hippocampus of the 6 LPS injections group (56.6 \pm 0.8 vs $46.7 \pm 1.9)$. Ferritin-positive cells increased similarly in the hippocampus of rats treated with a single (49.2 \pm 1.7 vs 28.1 $\pm 1.9)$ or repeated $(47.6 \pm 1.1$ vs $28.1 \pm 1.9)$ LPS dose. Single LPS enhanced Iba-1 in the paraventricular nucleus (PVN: 92.8 \pm 4.1 vs $65.2 \pm 2.2$ ) and hippocampus (99.4 \pm 4.4 vs $73.8 \pm 2.1$ ), but had no effect in the retrochiasmatic nucleus (RCA) and ARC. Interestingly, 6 LPS increased the lba-1 expression in these hypothalamic and hippocampal regions (RCA: $57.8 \pm 4.6 \mathrm{vs}$ $36.6 \pm 2.2$; ARC: $62.4 \pm 6.0$ vs $37.0 \pm 2.2 ;$ PVN: $100.7 \pm 4.4$ vs $65.2 \pm 2.2$; hippocampus: $123.0 \pm 3.8$ vs $73.8 \pm 2.1$ ). The results suggest that repeated LPS treatment stimulates the expression of glial activation markers, protecting neuronal activity during prolonged inflammatory challenges.
\end{abstract}

Key words: LPS tolerance; Astrocytes; Microglia; Glial fibrillary acidic protein; Iba-1; Glutamine synthetase; Ferritin

\section{Introduction}

Lipopolysaccharide (LPS), a Gram-negative bacterial cell surface component, has been used to stimulate the immune response both in peripheral tissues (1) and in the brain (2). It is well known that LPS increases the immunostaining for glial marker proteins, coupled with production of tumor necrosis factor- $\alpha$ (TNF- $\alpha)(3)$, interleukin-1 $\beta$ (IL-1 $\beta$ ) (4) and nitric oxide (5). In contrast, earlier reports demonstrated that long-term administration of LPS is accompanied by a tolerance to its effects, with desensitization of single LPS-induced responses (6-8). Nevertheless, little is known about the effect of chronic exposure to LPS on glial marker expression in vivo.

Reactive glia and the products induced by these cells are the most likely candidates for mediating inflammation in the brain. Upon activation, glial cells change their morphology and up-regulate the expression of a variety of cell-type selective markers, such as glial fibrillary acidic protein (GFAP) (2,9), glutamine synthetase (GS) (10), ionized calcium binding adaptor protein-1 (lba-1) $(11,12)$, and ferritin (13). Two groups of glial cells are presented in the brain: the macroglia, including astrocytes and oligodendrocytes, and the microglia. Astrocytes affect central nervous system (CNS) homeostasis through neurotrophic factor release that in turn affects neuronal function (14). Astrocytes undergo astrogliosis, characterized by an enhancement of GFAP expression in several neurodegenerative diseases (15). Astrogliosis can be a beneficial event to promote neuronal growth and survival, but it may also be damaging (16). However, the molecular mechanism underlying GFAP expression in activated astrocytes is poorly understood. Another important role of astrocytes is to take up excess ammonia and to convert glutamate into glutamine via the GS enzyme, protecting neurons against excitotoxicity (17). Thus, changes in GS expression may indicate alterations in astrocyte function. Of note, glial GS may also be involved in pathological brain conditions such

Correspondence: L.L.K. Elias, Departamento de Fisiologia, FMRP, USP, Av. Bandeirantes, 3900, 14049-900 Ribeirão Preto, SP, Brasil. Fax: +55-16-3633-0017. E-mail: llelias@fmrp.usp.br

Received October 11, 2011. Accepted April 19, 2012. Available online May 4, 2012. Published August 3, 2012. 
as Alzheimer's disease (18).

Some specific microglial markers are used to discriminate microglia from astrocytes. Imai et al. (19) isolated a rat cDNA clone encoding a novel gene designated iba1 (ionized calcium binding adapter molecule-1). The genomic copy of the iba1 gene is located within a segment of the major histocompatibility complex class III. Among brain cells, the iba1 gene was expressed specifically in the microglia cells and Iba-1 protein is suggested to act as an adaptor molecule, mediating calcium signaling in these cells. Reports have pointed out that active microglia and oligodendrocytes also express iron management proteins such as ferritin $(13,20)$. Ferritin peptide contains 24 subunits of heavy $(H)$ or light $(L)$ chain (21), which differ functionally. $\mathrm{H}$-ferritin converts soluble ferrous iron into the storable ferric form, while L-ferritin stores iron at a lower rate. Observations have suggested that the iron status of glial cells could influence physiological brain responses, since iron is essential for energy production, but may also generate reactive oxygen species (13).

A direct comparison of GFAP, GS, ferritin, and Iba-1 expression between acute and chronic experimental LPS infection in rodents is lacking. Therefore, in this study we investigated the expression of selected glial protein markers in the CNS of animals after single or repeated LPS exposure in order to clarify the role of glial cells in the mechanisms of tolerance to endotoxin.

\section{Material and Methods}

\section{Animals}

Male Wistar rats weighing 200-250 g (CentralAnimal Facility of Universidade de São Paulo, Ribeirão Preto Campus) were individually housed in a light- (12 h on, $12 \mathrm{~h}$ off) and temperature- $\left(23 \pm 1^{\circ} \mathrm{C}\right)$ controlled room with food and water available ad libitum. Rats were allowed to habituate to their environment for at least 3 days prior to the experimental procedures. During this period, rats were handled daily. All experimental protocols were approved by the Ethics Committee for Animal Use of Faculdade de Medicina de Ribeirão Preto.

\section{Animal treatment protocol}

The LPS from Escherichia coli used in this study was purchased from Sigma, USA (Serotype 026:B6). LPS was diluted in sterile saline $(0.15 \mathrm{M} \mathrm{NaCl})$ and injected ip at the dose of $100 \mu \mathrm{g} / \mathrm{kg}$, in a volume of $1 \mathrm{~mL} / \mathrm{kg}$. The LPS or saline $(1 \mathrm{~mL} / \mathrm{kg}$ ) injection was made between 4:00 and 4:30 pm.

\section{Experimental procedure}

GFAP, GS, ferritin, and the Iba-1 protein immunostaining after single or repeated LPS injections. For the experiments, rats were assigned to three different groups of ip treatment ( $N=5-6$, per group): 1) saline once daily for 6 days (6 saline); 2) saline once daily for 5 days and an injection of LPS on the 6 th day ( 5 saline +1 LPS), and 3) LPS once daily for 6 days (6 LPS).
Twenty-four hours after the last injection, animals were anesthetized with an overdose of 2,2,2-tribromoethanol (2.5\%, $1 \mathrm{~mL} / 100 \mathrm{~g} \mathrm{ip}$; Aldrich, USA) and transcardially perfused first with $200 \mathrm{~mL}$ saline, followed by $300 \mathrm{~mL} 4 \%$ formaldehyde in $0.1 \mathrm{M}$ phosphate buffer. The brain was collected, postfixed in the same fixative for $24 \mathrm{~h}$, placed in PBS containing $30 \%$ sucrose, and stored at $4^{\circ} \mathrm{C}$.

\section{Immunohistochemistry}

Coronal brain sections were cut at $30 \mu \mathrm{m}$ thickness and free-floating preserved in cryoprotectant at $-20^{\circ} \mathrm{C}$. One of every fifth section was used for each glial marker immunostaining. Briefly, after removal of the sections from the cryoprotectant and rinsing in $50 \mathrm{mM} \mathrm{KPBS}, \mathrm{pH} 7.4$, sections were incubated for $1 \mathrm{~h}$ at room temperature, then for $48 \mathrm{~h}$ at $4^{\circ} \mathrm{C}$ with primary antibody rabbit anti-GFAP (monoclonal, 1:1000; Zymed Laboratories, USA) or mouse anti-GS (monoclonal, 1:500; Chemicon, USA) or rabbit anti-lba-1 (monoclonal, 1:5000, Wako Chemicals, USA) or human anti-ferritin (polyclonal, 1:5000, Sigma). After rinsing, sections were incubated for $1 \mathrm{~h}$ with biotinylated secondary goat anti-rabbit antibody for GFAP (1:600) and Iba-1 (1:200), rabbit anti-human antibody for ferritin (1:200), or goat anti-mouse antibody for GS (1:200) (Vector Laboratories, USA), and processed by the Vectastatin Elite avidin-biotin immunoperoxidase method. Solutions of diaminobenzidine, nickel sulfate, and $\mathrm{H}_{2} \mathrm{O}_{2}$ were used to generate blue-black immunolabeling. We also performed immunostaining with the primary antibodies described above and the secondary donkey anti-mouse CY5 antibody (1:500, Jackson, USA) or FITC donkey anti-rabbit antibody (1:500, Jackson) for qualitative immunofluorescence.

The photomicrographs were captured with a Leica microscope equipped with a DC 200 digital camera, attached to a contrast enhancement device. The immunoreactive-positive cells of three sections per rat were estimated by counting black staining from an area at the level of the paraventricular (PVN), arcuate (ARC) and retrochiasmatic (RCA) nuclei in the hypothalamus and hippocampus, using the Image $J^{\circledR}$ software (Version 1.38, NIH, USA). We considered the coordinates supplied by Paxinos and Watson atlas (22): PVN and RCA at level $-1.80 \mathrm{~mm}$ from bregma and ARC and hippocampus at level -2.12 to $-3.30 \mathrm{~mm}$ from bregma.

\section{Statistical analysis}

Data are reported as means \pm SEM. One-way ANOVA followed by the Bonferroni post hoc test was used for analysis of the number of positive immunolabeled cells. Significance was set at $P<0.05$.

\section{Results}

\section{Expression of GFAP in the hypothalamus and hippocampus after single or repeated LPS injection}

A single dose of LPS induced a significant increase in the number of GFAP-positive cells compared to 6 saline- 
treated rats in the ARC and in the hippocampus (Figure 1). Repeated exposure to LPS induced a further enhancement $(P<0.05)$ of GFAP-positive cells in the ARC and hippocampus compared to 6 saline- or single LPS-treated animals. There was no difference in GFAP-positive cells in the PVN between the saline and LPS-treated groups.

\section{Expression of GS in the hippocampus after single or repeated LPS injections}

A single dose of LPS induced no alteration in the number of GS-positive cells in the hippocampus compared to 6 saline rats (Figure 2). On the other hand, 6 doses of LPS increased $(P<0.05)$ the number of $G S$-positive cells in the hippocampus compared to controls and to the single LPS treatment. No changes in GS immunostaining were observed in any other brain regions investigated.

\section{Expression of ferritin in the hippocampus after single or repeated LPS injections}

We observed that a single LPS injection and also 6 LPS injections induced a significant increase $(P<0.05)$ in the number of ferritin-positive cells in the hippocampus (Figure 3) compared to 6 saline-treated rats. We did not observe changes in ferritin immunostaining in the hypothalamus.

\section{Expression of Iba-1 in the hypothalamus and hippocampus after single or repeated LPS injections}

Our results show that a single dose of LPS increased $(P<0.05)$ the number of Iba-1-positive cells compared to 6 saline rats, only in the PVN and hippocampus (Figures 4 and 5). On the other hand, repeated LPS injections increased ( $P$ $<0.05$ ) the Iba-1-expressing cells in the RCA, ARC, PVN, and hippocampus compared to control rats.

\section{Discussion}

In the present study, $24 \mathrm{~h}$ after a single exposure to LPS, animals showed a higher number of GFAP-, ferritin- and Iba-1-positive cells in the hippocampus and GFAP- and Iba-1-positive cells in the hypothalamus, with no change in
A

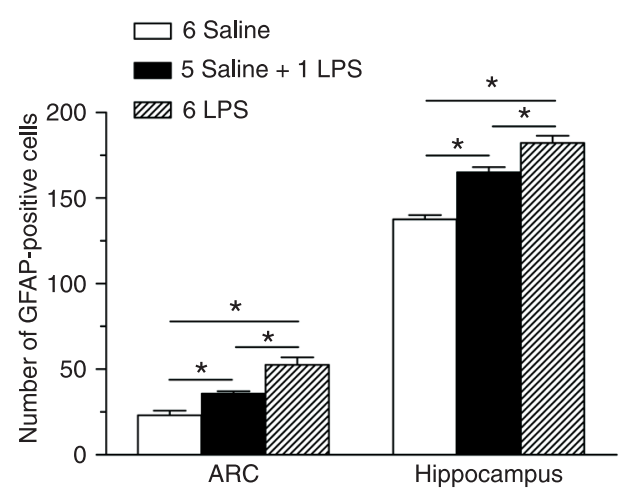

B

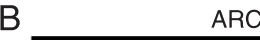

ARC
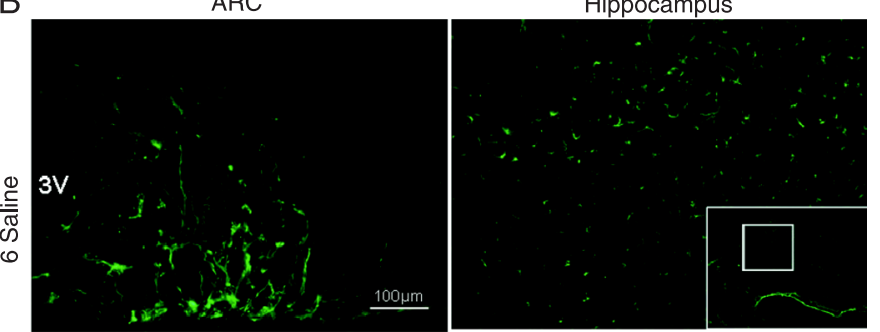

מ

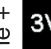

$3 \mathrm{~V}$
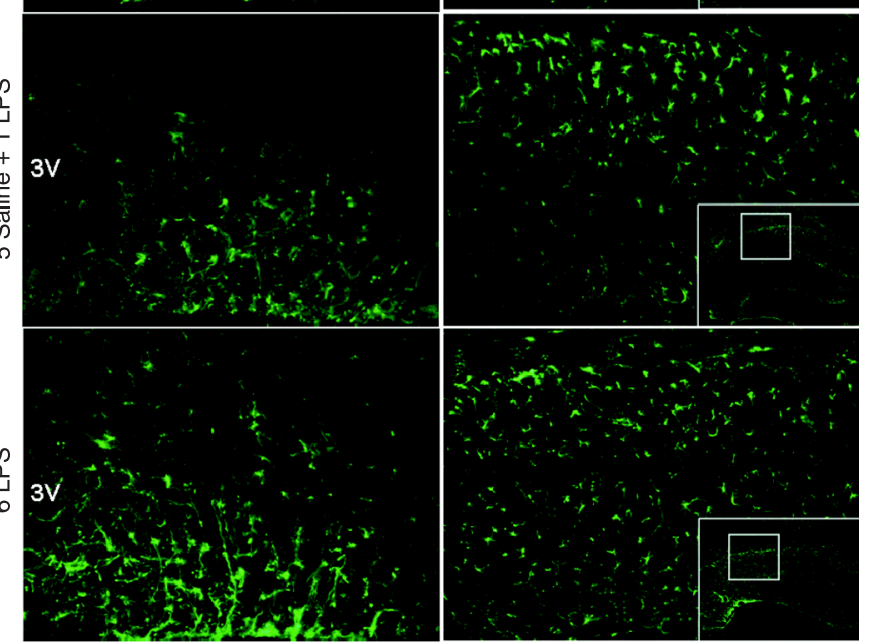

Figure 1. Panel A, Number of glial fibrillary acidic protein (GFAP)-positive cells in the arcuate (ARC) nucleus and hippocampus of animals injected with 6 doses of saline (6 saline), a single dose of lipopolysaccharide (LPS; 5 saline +1 LPS) or repeated doses of LPS (6 LPS). Panel B, Representative photomicrographs showing the distribution of astrocyte GFAP immunostaining (green) in the medial portion of the ARC nucleus (left column) and hippocampus (right column) in animals injected with 6 doses of saline (6 saline), a single dose of LPS ( 5 saline +1 LPS) or repeated doses of LPS ( 6 LPS). Insets show the areas where the photomicrographs were taken at higher magnification $(100 \mathrm{X}) .3 \mathrm{~V}=$ third ventricle. Data are reported as means \pm SEM and are representative of two independent experiments $(N=5-6)$. ${ }^{*} P<0.05$ (one-way ANOVA, followed by the Bonferroni post hoc test). 


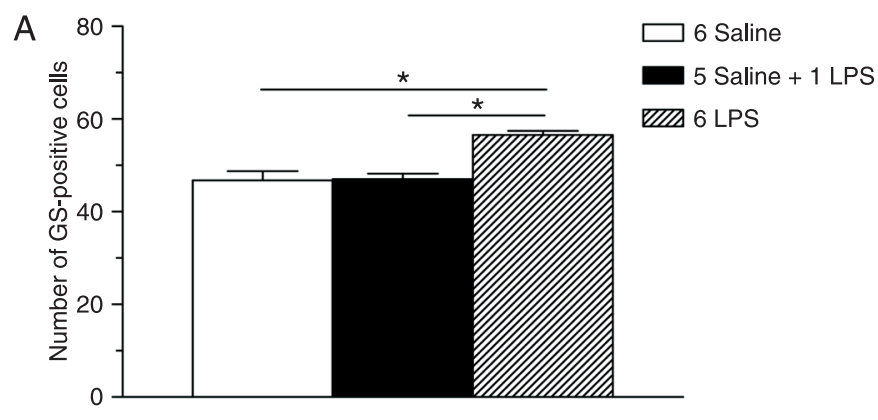

B
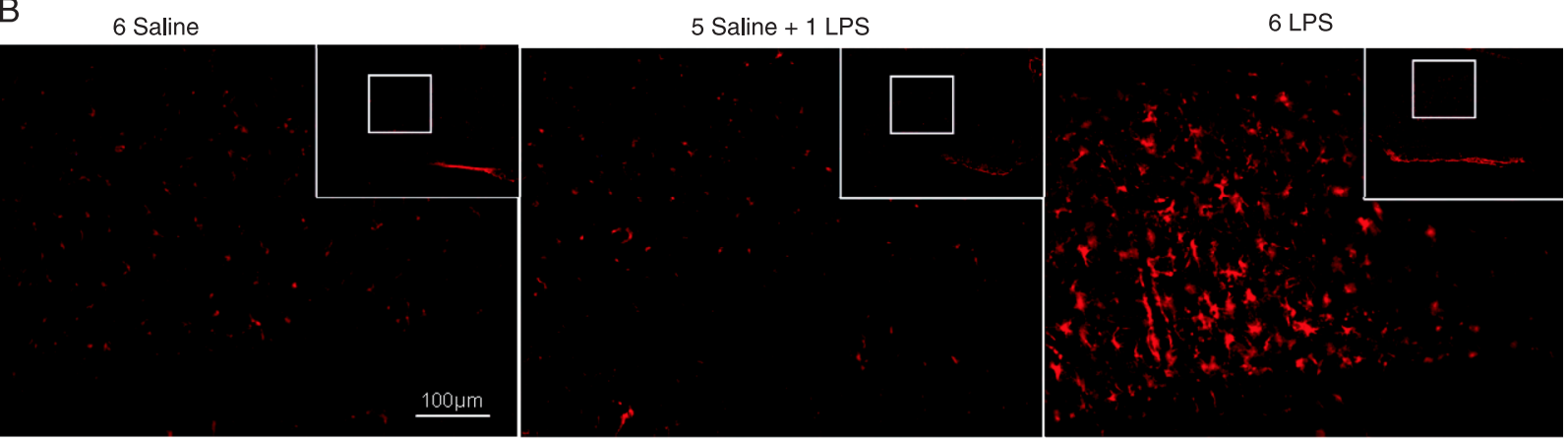

Figure 2. Panel $A$, Graph showing the number of glutamine synthetase (GS)-positive cells in the hippocampus of animals injected with 6 doses of saline (6 saline), a single dose of lipopolysaccharide (LPS; 5 saline +1 LPS) or repeated doses of LPS (6 LPS). Panel B, Representative photomicrographs showing the distribution of glial GS immunostaining (red) in the hippocampus. Insets show the areas where the photomicrographs were taken at higher magnification (100X). Data are reported as means \pm SEM and are representative of two independent experiments $(\mathrm{N}=5-6)$. ${ }^{*} \mathrm{P}<0.05$ (one-way ANOVA, followed by the Bonferroni post hoc test).

the number of GS-positive cells. Repeated LPS stimulation induced a further increase in GFAP and Iba-1 expression in the hypothalamus and hippocampus. We also found that repeated LPS treatment induced a marked enhancement of GS and ferritin expression in the hippocampus compared to controls.

GFAP seems to have specific patterns of up-regulation induced by systemic LPS injection, as we observed both in the hypothalamic ARC and hippocampus. Similarly, Herber et al. (2) showed an enrichment of astrogliosis in the hippocampus after acute centrally injected LPS. In our results, long-term exposure to LPS exacerbated the astrogliosis both in the hypothalamus and hippocampus, showing no desensitization of astrocytic responses following repeated LPS injections. Although the exact mechanism of GFAP stimulation remains unclear, our data are consistent with the well-recognized function of astrocytes in immune and inflammatory challenges in the CNS $(14,23)$. Astrocytes account for the improvement of the number of functional synapses in the CNS (24) and this could be important to maintain the actions of inflammatory mediators during both acute and chronic endotoxemia. Moreover, astrocytes contribute to the structural and functional integrity of the blood brain barrier (BBB) (14). Our observation of marked
GFAP expression in the ARC suggests that astrocytes could also preserve the function of regions close to the median eminence, in which BBB is not present, under acute or frequent LPS exposure.

We observed for the first time that after recurrent LPS treatment in rodents there is a remarkable enhancement of GS expression in the hippocampus, in contrast to single LPS treatment. Studies have pointed out that excessive production of glutamate, an excitatory amino acid neurotransmitter, induces neurodegeneration and damage in neurologic function $(25,26)$ and astrocytes seems to protect neurons against glutamate-induced neurotoxicity by the expression of GS (27). Therefore, the increased GS expression in the hippocampus may be a neuroprotective mechanism during repeated exposure to endotoxin. In vivo acute LPS increased GS activity by $250 \%$ in adult rats (28). However, it has been shown that in primary astroglial cultures LPS down-regulated GS-mRNA and GS expression with a transitory increase in IL-1 $\beta$ mRNA expression (29). Chao et al. (27) observed that TNF- $\alpha$ inhibited GS activity in mixed neuronal/astrocyte and enriched astrocyte cultures. We previously observed that acute LPS treatment increased plasma TNF- $\alpha$, and rats under repeated LPS stimulation showed desensitization of the TNF- $\alpha$ response, with no TNF- $\alpha$ enhancement (7). The 


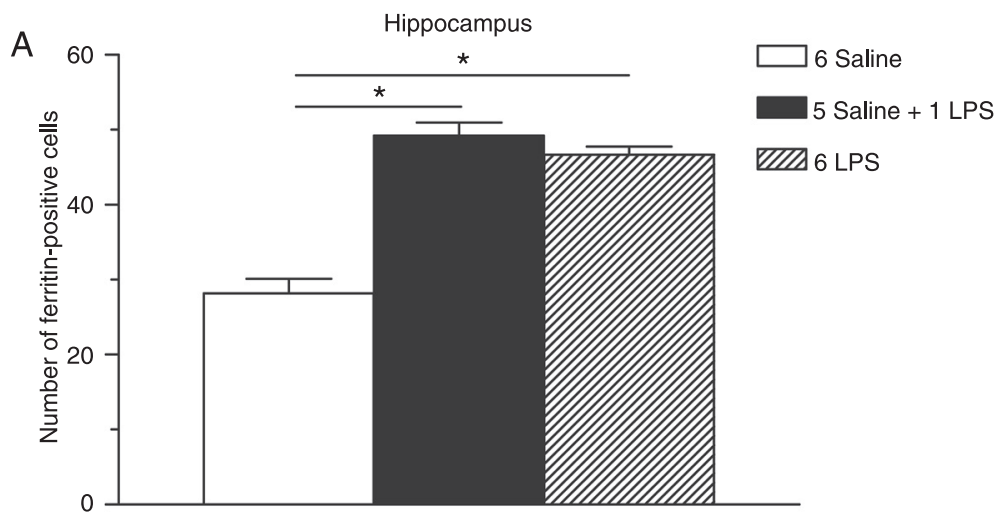

B
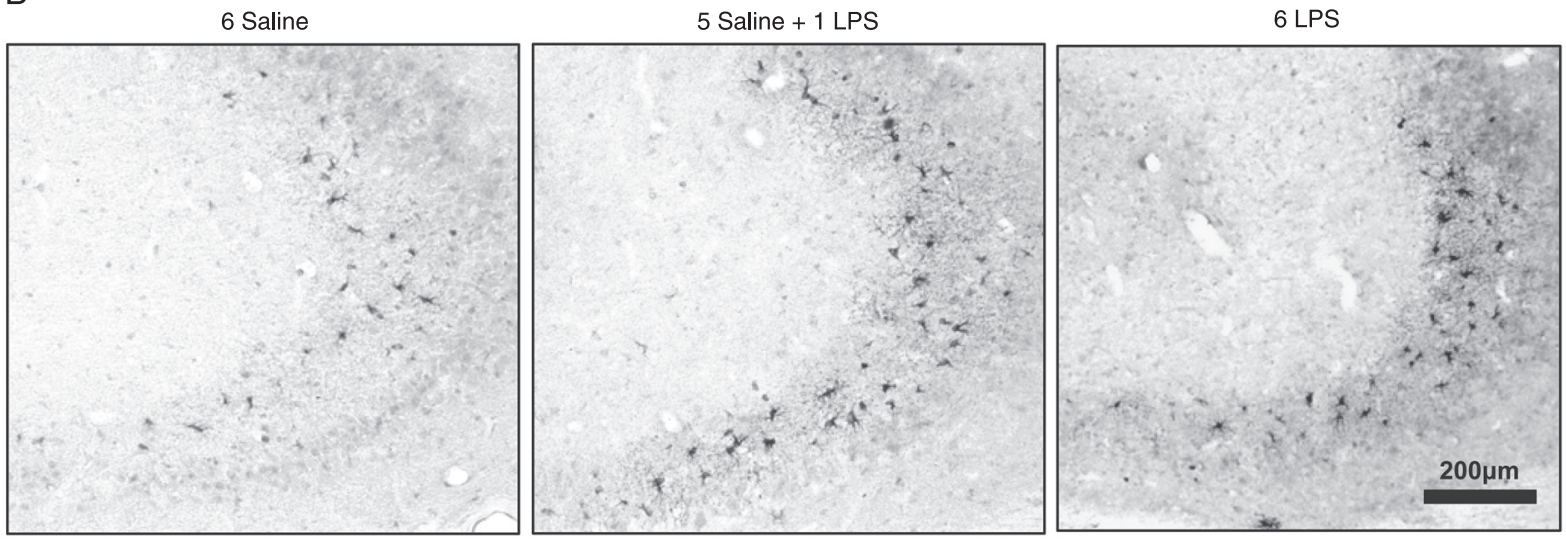

Figure 3. Panel A, Graph showing the number of ferritin-positive cells in the hippocampus of animals injected with 6 doses of saline (6 saline), a single dose of lipopolysaccharide (LPS; 5 saline + 1 LPS) or repeated doses of LPS (6 LPS). Panel B, Representative bright field photomicrographs showing the distribution of ferritin immunostaining (black staining). Data are reported as means \pm SEM and are representative of two independent experiments $(N=5-6)$. ${ }^{*} P<0.05$ (one-way ANOVA, followed by the Bonferroni post hoc test).

absence of alteration in GS expression in the single LPS group could be, at least in part, a result of TNF- $\alpha$ inhibition. Furthermore, glial cells can produce glutamine synthetase in response to several stimuli such as insulin, corticosteroid hormones, thyroid hormones, and hyperammonemic conditions (10), and these stimuli are affected by LPS.

We observed in the hippocampus a large number of ferritin-expressing cells after single and repeated LPS injection. Inflammation affects iron metabolism and many inflammatory mediators act via iron-responsive proteins. Ferritin was primarily considered to be an iron storage protein, and recently it was demonstrated that it can deliver iron to multiple organs, including the brain (30). It is known that inflammatory conditions decrease iron levels in blood (31) due to retention of iron within the cells. This phenomenon could be a defense reaction to deprive invading pathogens of iron, in parallel to an antioxidant defense. Duvigneau et al. (32) reported that iron metabolism is expected to be modulated in LPS-induced septic shock, since cytokine and nitric oxide are produced upon LPS stimulation, and both

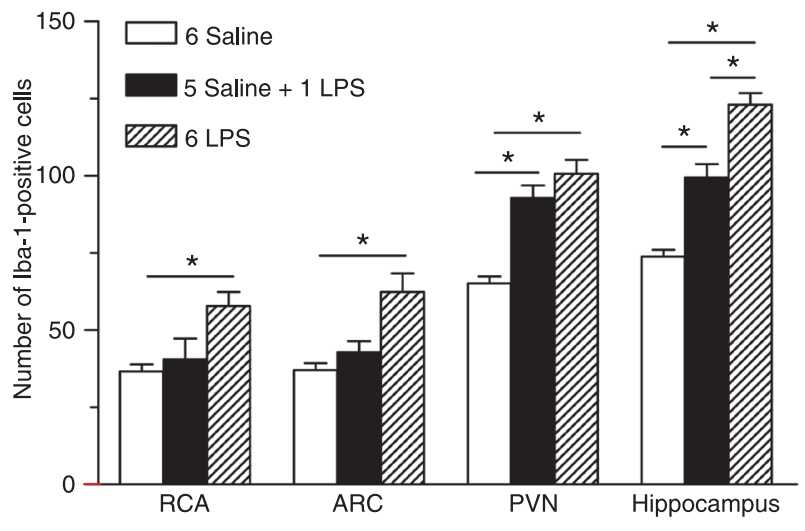

Figure 4. Number of ionized calcium binding adaptor protein-1 (lba-1)-positive cells in the retrochiasmatic (RCA), arcuate (ARC) and paraventricular (PVN) nuclei, and hippocampus of animals injected with 6 doses of saline ( 6 saline), a single dose of lipopolysaccharide (LPS; 5 saline +1 LPS) or repeated doses of LPS (6 LPS). Data are reported as means \pm SEM and are representative of two independent experiments $(\mathrm{N}=5-6)$. ${ }^{*} \mathrm{P}<0.05$ (one-way ANOVA, followed by the Bonferroni post hoc test). 


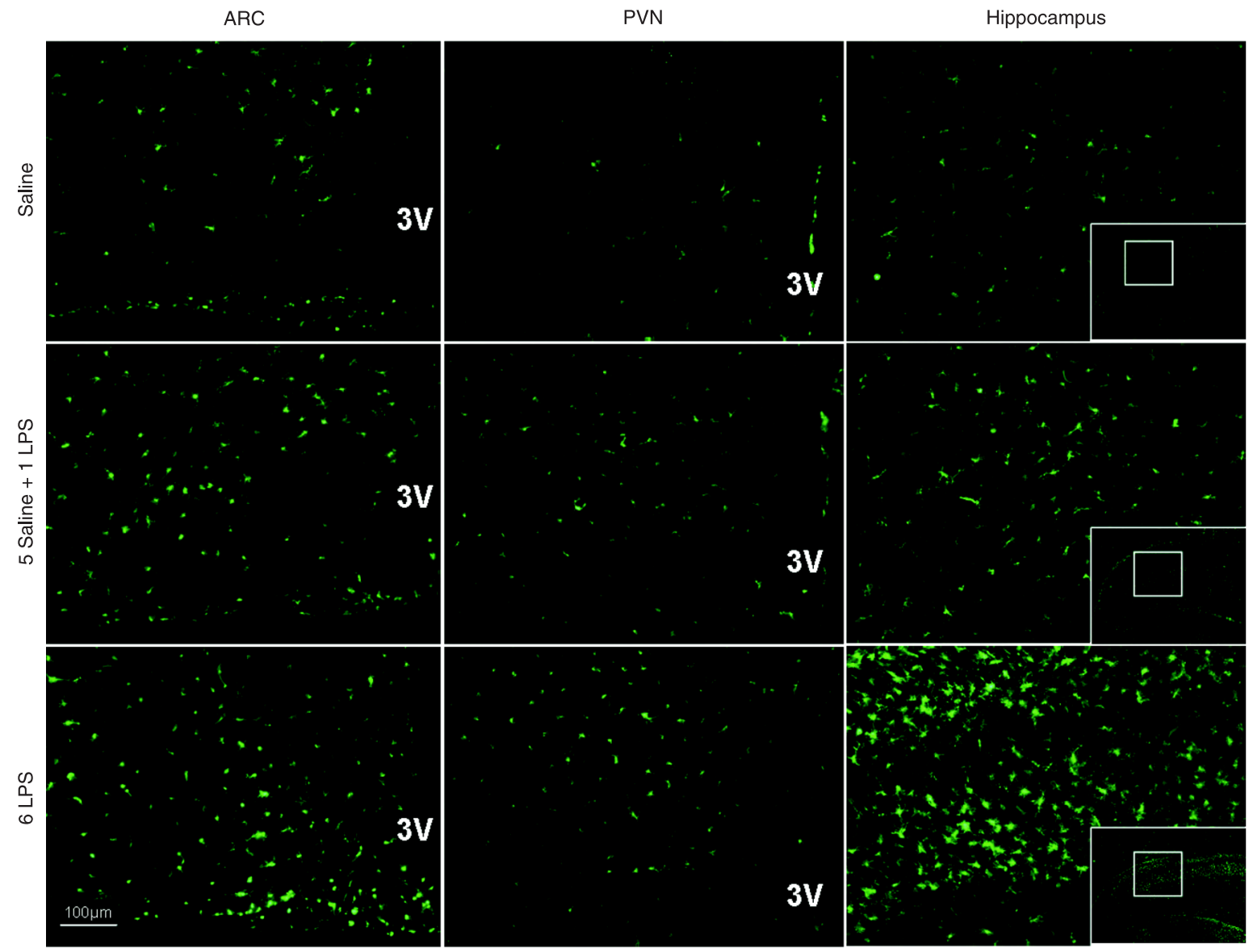

Figure 5. Representative photomicrographs showing the distribution of microglial ionized calcium binding adaptor protein-1 (Iba-1) immunostaining (green) in the medial portion of the arcuate nucleus (ARC; left column), paraventricular nucleus (PVN; middle column) and hippocampus (right column) of animals injected with 6 doses of saline (6 saline), a single dose of lipopolysaccharide (LPS; 5 saline +1 LPS) or repeated doses of LPS (6 LPS). Insets show the areas where the photomicrographs were taken at higher magnification (100X). $3 \mathrm{~V}=$ third ventricle

of them could influence iron-responsive proteins. Zhang et al. (1) showed that injection of LPS into the globus pallidus of rats substantially increased iron levels in glial cells of the substantia nigra pars reticulata, suggesting that LPS inflammation leads to stress of dopamine-containing neurons, increasing brain vulnerability to degenerative processes. The enhancement of ferritin expression in animals with short- or long-term exposure to LPS observed in our study could account for a protective effect on the CNS.

Our observations indicate an increased number of cells immunoreactive to the Iba-1 protein in the hypothalamus and hippocampus in response to acute LPS. For the first time, we showed a higher number of Iba-1-positive cells in these regions elicited by repeated LPS treatment. Microglia in the CNS were found to respond rapidly to pathogens and also to be involved in several components of the neuroinflammatory response. Our data regarding LPS induction of Iba-1 are consistent with published reports (11), and the present study adds further evidence of microglia participation in response to inflammation, as shown by the enhancement of Iba-1 expression during prolonged inflammatory challenge. The contribution of microglia to the modulation of neurogenesis under pathological conditions has both pro- and anti-neurogenic effects. Cacci et al. (33) demonstrated that acutely activated microglia prevented neuronal differentiation and strongly increased glial differentiation mediated by proinflammatory cytokines, whereas chronically activated microglia were shown to be permissive to neuronal differentiation and cell survival with preserved glial differentiation. These data suggest that, in a chronically altered environment, persistently activated microglia can have protective functions that favor rather than hinder brain repair processes.

Glial cells are known to regulate innate immunity and participate in adaptive immune responses in CNS tissue. In response to injury, ischemia and inflammatory stimuli, 
glial cells may have an activated phenotype associated with proliferation, migration to the site of injury and secretion of both neurotoxic and neurotrophic factors. Chen et al. (34), using short- or long-course LPS treatment with either low $(250 \mu \mathrm{g} / \mathrm{kg})$ or high $(32 \mathrm{mg} / \mathrm{kg})$ LPS dose, found that during repeated exposure to endotoxin, LPS-induced TNF- $\alpha$, IL-1 $\beta$ and IL- 6 expression was sustained in the brain even though cytokines were no longer induced at the periphery. Our findings of a higher expression of glial markers are therefore in line with the sustained brain cytokine levels observed after long-course LPS treatment. To verify the role of glial activity and its relationship with the severity of systemic endotoxemia, further studies will be necessary to investigate the effects of high and low doses of LPS on expression of markers of glia activity

During long-term LPS endotoxemia, although several hormonal and peripheral cytokine responses show the

\section{References}

1. Zhang J, Stanton DM, Nguyen XV, Liu M, Zhang Z, Gash $D$, et al. Intrapallidal lipopolysaccharide injection increases iron and ferritin levels in glia of the rat substantia nigra and induces locomotor deficits. Neuroscience 2005; 135: 829838.

2. Herber DL, Maloney JL, Roth LM, Freeman MJ, Morgan $D$, Gordon MN. Diverse microglial responses after intrahippocampal administration of lipopolysaccharide. Glia 2006; 53: 382-391.

3. Sawada M, Kondo N, Suzumura A, Marunouchi T. Production of tumor necrosis factor-alpha by microglia and astrocytes in culture. Brain Res 1989; 491: 394-397.

4. Hetier E, Ayala J, Denefle P, Bousseau A, Rouget P, Mallat $M$, et al. Brain macrophages synthesize interleukin-1 and interleukin-1 mRNAs in vitro. J Neurosci Res 1988; 21: 391397.

5. Lee P, Son D, Lee J, Kim YS, Kim H, Kim SY. Excessive production of nitric oxide induces the neuronal cell death in lipopolysaccharide-treated rat hippocampal slice culture. Neurosci Lett 2003; 349: 33-36.

6. Ziegler-Heitbrock HW. Molecular mechanism in tolerance to lipopolysaccharide. J Inflamm 1995; 45: 13-26.

7. Borges BC, Antunes-Rodrigues J, Castro M, Bittencourt JC, Elias CF, Elias LL. Expression of hypothalamic neuropeptides and the desensitization of pituitary-adrenal axis and hypophagia in the endotoxin tolerance. Horm Behav 2007; 52: 508-519.

8. Borges BC, Rorato R, Avraham Y, da Silva LE, Castro M, Vorobiav $\mathrm{L}$, et al. Leptin resistance and desensitization of hypophagia during prolonged inflammatory challenge. $\mathrm{Am}$ J Physiol Endocrinol Metab 2011; 300: E858-E869.

9. Guo LH, Schluesener HJ. Acute but not chronic stimulation of glial cells in rat spinal cord by systemic injection of lipopolysaccharide is associated with hyperalgesia. Acta Neuropathol 2006; 112: 703-713.

10. Frank MG, Baratta MV, Sprunger DB, Watkins LR, Maier SF. Microglia serve as a neuroimmune substrate for stressinduced potentiation of CNS pro-inflammatory cytokine phenomenon of endotoxin tolerance, the expression of different glial markers is not desensitized, indicating an enhanced activation of astrocytes and microglia in different CNS regions. The maintenance of glial cell responsiveness to endotoxin may contribute to the maintenance of CNS functions, thus preserving host survival.

\section{Acknowledgments}

We would like to thank Dr. Gloria Hoffman (Morgan State University, USA) for the immunohistochemistry protocol. The authors are grateful to Maria Valci Aparecida dos Santos Silva and Milene Mantovani (Faculdade de Medicina de Ribeirão Preto, Universidade de São Paulo, Brazil) for excellent technical assistance. We also wish to thank FAPESP, FAEPA, and CNPq for financial support.

responses. Brain Behav Immun 2007; 21: 47-59.

11. Ji KA, Yang MS, Jeong HK, Min KJ, Kang SH, Jou I, et al. Resident microglia die and infiltrated neutrophils and monocytes become major inflammatory cells in lipopolysaccharide-injected brain. Glia 2007; 55: 1577-1588.

12. Romero-Sandoval A, Chai N, Nutile-McMenemy N, Deleo JA. A comparison of spinal lba1 and GFAP expression in rodent models of acute and chronic pain. Brain Res 2008; 1219: 116-126.

13. Zhang $X$, Surguladze N, Slagle-Webb B, Cozzi A, Connor JR. Cellular iron status influences the functional relationship between microglia and oligodendrocytes. Glia 2006; 54: 795-804.

14. Dong Y, Benveniste EN. Immune function of astrocytes. Glia 2001; 36: 180-190.

15. Hatten ME, Liem RK, Shelanski ML, Mason CA. Astroglia in CNS injury. Glia 1991; 4: 233-243.

16. Eng LF, Ghirnikar RS. GFAP and astrogliosis. Brain Pathol 1994; 4: 229-237.

17. Derouiche A, Ohm TG. Glutamine synthetase immunoreactivity in the human hippocampus is lamina-specific. Neurosci Lett 1994; 165: 179-182.

18. Robinson SR. Neuronal expression of glutamine synthetase in Alzheimer's disease indicates a profound impairment of metabolic interactions with astrocytes. Neurochem Int 2000; 36: 471-482.

19. Imai $Y$, Ibata I, Ito D, Ohsawa K, Kohsaka S. A novel gene iba1 in the major histocompatibility complex class III region encoding an EF hand protein expressed in a monocytic lineage. Biochem Biophys Res Commun 1996; 224: 855862.

20. Qi Y, Jamindar TM, Dawson G. Hypoxia alters iron homeostasis and induces ferritin synthesis in oligodendrocytes. $J$ Neurochem 1995; 64: 2458-2464.

21. Levi S, Yewdall SJ, Harrison PM, Santambrogio P, Cozzi A, Rovida $\mathrm{E}$, et al. Evidence of $\mathrm{H}$ - and L-chains have cooperative roles in the iron-uptake mechanism of human ferritin. Biochem J 1992; 288 (Part 2): 591-596. 
22. Paxinos G, Watson C. The rat brain in stereotaxic coordinates. San Diego: Academic Press; 1998.

23. Aschner M. Astrocytes as mediators of immune and inflammatory responses in the CNS. Neurotoxicology 1998; 19: 269-281.

24. Ullian EM, Sapperstein SK, Christopherson KS, Barres BA. Control of synapse number by glia. Science 2001; 291: 657661.

25. Choi DW. Glutamate neurotoxicity and diseases of the nervous system. Neuron 1988; 1: 623-634.

26. Cotman CW, Lynch GS. The neurobiology of learning and memory. Cognition 1989; 33: 201-241.

27. Chao CC, Hu S, Tsang M, Weatherbee J, Molitor TW, Anderson WR, et al. Effects of transforming growth factor-beta on murine astrocyte glutamine synthetase activity. Implications in neuronal injury. J Clin Invest 1992; 90: 1786-1793.

28. Sarantos P, Abouhamze A, Chakrabarti R, Souba WW. Glucocorticoids regulate intestinal glutamine synthetase gene expression in endotoxemia. Arch Surg 1994; 129: 59-65.

29. Letournel-Boulland ML, Fages C, Rolland B, Tardy M. Lipopolysaccharides (LPS), up-regulate the IL-1-mRNA and down-regulate the glial fibrillary acidic protein (GFAP) and glutamine synthetase (GS)-mRNAs in astroglial primary cultures. Eur Cytokine Netw 1994; 5: 51-56.

30. Fisher J, Devraj K, Ingram J, Slagle-Webb B, Madhankumar $A B$, Liu $X$, et al. Ferritin: a novel mechanism for delivery of iron to the brain and other organs. Am J Physiol Cell Physiol 2007; 293: C641-C649.

31. Weiss $\mathrm{G}$. Modification of iron regulation by the inflammatory response. Best Pract Res Clin Haematol 2005; 18: 183201.

32. Duvigneau JC, Piskernik C, Haindl S, Kloesch B, Hartl RT, Huttemann $M$, et al. A novel endotoxin-induced pathway: upregulation of heme oxygenase 1 , accumulation of free iron, and free iron-mediated mitochondrial dysfunction. Lab Invest 2008; 88: 70-77.

33. Cacci E, Ajmone-Cat MA, Anelli T, Biagioni S, Minghetti L. In vitro neuronal and glial differentiation from embryonic or adult neural precursor cells are differently affected by chronic or acute activation of microglia. Glia 2008; 56: 412425. 